\title{
Macroporous Platinum Electrodes for Hydrogen Oxidation in Ionic Liquids
}

\author{
Ghulam Hussain, ${ }^{1}$ M. Veronica Sofianos, ${ }^{2}$ Junqiao Lee, ${ }^{1}$ Caitlyn Gibson, ${ }^{1}$ Craig E. \\ Buckley $^{2}$ and Debbie S. Silvester*1
}

\author{
${ }^{1}$ Curtin Institute for Functional Molecules and Interfaces \& Department of Chemistry, Curtin University, GPO \\ Box U1987, Perth, 6845, Australia. \\ ${ }^{2}$ Department of Physics and Astronomy, Curtin University, GPO Box U1987, Perth, WA 6845, Australia. \\ *E-mail: d.silvester-dean@ curtin.edu.au; Fax: +61(8)92664699; Tel: +61(8)92667148
}

\begin{abstract}
Macroporous platinum structures have been prepared by electrodeposition in the interstitial spaces between a $500 \mathrm{~nm}$ polystyrene sphere template, onto platinum and glassy carbon electrodes. The structures were analysed with scanning electron microscopy and confocal microscopy. These electrodes are employed for the electrochemical oxidation of hydrogen in the room temperature ionic liquid 1-ethyl-3-methylimidazolium bis(trifluoromethylsulfonyl)imide $\quad\left(\left[\mathrm{C}_{2}\right.\right.$ mim] $\left.]\left[\mathrm{NTf}_{2}\right]\right)$. The behaviour on the porous electrodes showed obvious thin-layer characteristics in the cyclic voltammetry, with a strong tendency for hydrogen to accumulate and remain in the pores after being removed from the cell. Plots of peak current vs concentration $\left(10-100 \% \mathrm{H}_{2}\right)$ were linear, but currents continued to increase over time. The sensitivities (gradients) of the calibration plots were the highest for the platinum porous structures (compared to the bare, or nanoparticle-modified surfaces). Due to the accumulation of gas, such modified electrodes could be employed as leak-detectors for very low concentrations of hydrogen.
\end{abstract}

\section{Keywords}

Macroporous platinum; electrodeposition; gas sensing; hydrogen oxidation; voltammetry; room temperature ionic liquid. 


\section{Introduction}

Hydrogen is an important, widely-used gas that finds applications in a range of fields, including as a rocket fuel during aerospace operations [1], for removing sulphur from fuels during oil-refining [2], as a reagent to synthesise ammonia in the Haber process [3], for removing friction-heat in power plant turbines [2], as a therapeutic medical gas [4], and it is a product in many chemical reactions. It is also being used as a fuel for next-generation hydrogen powered vehicles, since the only emission products are water [5]. It is colourless and odourless but presents a potential hazard, since it forms explosive mixtures with air in the concentration range $4-75 \%$ vol. Continuous monitoring for potential leaks and build-up of hydrogen is therefore required, and electrochemical hydrogen sensors have been developed to overcome this challenge [6].

In addition to detecting hydrogen, it is also important to understand the hydrogen oxidation reaction (HOR) in fuel cells. It is well known that the mechanism for HOR in aqueous solutions involves the formation of adsorbed hydrogen on platinum, followed by its dissociative oxidation $[7,8]$ :

$$
\mathrm{Pt}+\frac{1}{2} \mathrm{H}_{2(\mathrm{~g})} \rightleftharpoons \mathrm{Pt}-\mathrm{H}_{(\mathrm{ad})} \rightleftharpoons \mathrm{Pt}+\mathrm{H}^{+}+e^{-}
$$

Less has been published on the mechanism in aprotic solvents such as room temperature ionic liquids (RTILs). The oxidation of hydrogen on Pt in RTILs has been reported by various groups [9-19], with pre-anodization ("activation") of the electrode giving rise to higher currents and more reversible voltammetry $[9,12,15,16]$. A detailed four-step mechanism involving the radical of the RTIL anion $\left(\left[\mathrm{NTf}_{2}\right]^{-}\right)$was recently proposed [17]:

$$
\begin{gathered}
\mathrm{NTf}_{2}^{-} \rightleftharpoons \mathrm{NTf}_{2}{ }^{\cdot}+e^{-} \\
\text {Pt-NTf }_{2}^{-} \rightleftharpoons \mathrm{Pt}-N T f_{2}{ }^{-}+e^{-} \\
2 \text { Pt-NTf } \\
{ }^{\circ}+\mathrm{H}_{2} \rightleftharpoons 2 \text { H-Pt-NTf } \\
\text { H-Pt-NTf } \\
2
\end{gathered}
$$


In an effort to explore more sensitive electrode materials for gas sensing applications, high surface area porous electrodes were prepared in this work. The desired physical effects of these porous materials that may lead to advantageous sensing properties are: (i) a larger surface area to enable a greater preconcentration of hydrogen at the electrode via adsorption, and (ii) "thin-layer" adsorption of hydrogen within the porous electrode - allowing quantification of the amount of hydrogen initially located in the porous layer. Porous electrodes of various materials (e.g. gold, platinum, palladium, cobalt, aluminium) have previously been produced by template-assisted electrodeposition [20-25], and some were used for electroanalytical applications $[25,26]$. In the present work, we report the preparation of platinum modified electrodes for the targeted detection of hydrogen gas in a RTIL. This is the first time that such porous electrode materials have been employed for ambient temperature (ca. 298 K) gas sensing using RTILs.

\section{Experimental}

Electrochemical experiments were preformed using a PGSTAT101 (Metrohm Autolab, Netherlands) interfaced to a PC with NOVA 1.11 software. For deposition experiments, a three-electrode cell was employed; the working electrode was a "change-disk" platinum (Pt) or glassy carbon (GC) stub $5 \mathrm{~mm}$ in diameter (PINE Research Instrumentation Inc., Durham, $\mathrm{NC}, \mathrm{USA}$ ), with a $\mathrm{Ag} / \mathrm{AgCl}$ reference electrode (BASi, Indiana, USA) and Pt coil counter electrode (Goodfellow, UK). The electrodes were immersed into $10 \mathrm{~mL}$ of electrolyte solution in a glass vial. Ultrapure water (resistance $18.2 \mathrm{M} \Omega \mathrm{cm}$ ) was prepared by an ultrapure water purification system (Millipore Pty Ltd., North Ryde, NSW, Australia). The working electrodes were first polished on soft lapping pads (Buehler Illinois) with decreasing size of alumina (3,1 and $0.5 \mu \mathrm{m}$, Kemet, NSW, Australia). For deposition, GC and Pt electrodes were templated with polystyrene latex spheres (500 nm diameter, $10 \mathrm{wt} \%$ aqueous suspension, Magsphere Inc., Pasadena California) in a 1:3 water:ethanol preparation. $5 \mu \mathrm{L}$ of

polystyrene sphere suspension was spread over the electrode, covered, and allowed to dry for ca. 4 hours. Electrodeposition was performed in $5 \mathrm{mM}$ chloroplatinic acid hydrate $\left(\mathrm{H}_{2} \mathrm{PtCl}_{6} \cdot \mathrm{xH}_{2} \mathrm{O}, \geq 99.9 \%\right.$, 
Sigma-Aldrich) $0.5 \mathrm{M} \mathrm{H}_{2} \mathrm{SO}_{4(\mathrm{aq})}(98 \% \mathrm{w} / \mathrm{w}$ [18.4 M], Sigma-Aldrich) solution, by holding at $-0.2 \mathrm{~V}$ (vs $\mathrm{Ag} / \mathrm{AgCl}$ ) for 300 seconds. After deposition, the electrodes were washed with ultrapure water, dried under nitrogen, before immersing in tetrahydrofuran ( $\geq 99.9 \%$, Sigma-Aldrich) for 2 days to dissolve the polystyrene spheres, and finally washed with isopropanol (99.5\%, Sigma-Aldrich).

For gas sensing experiments, the reference and counter electrodes were Ag and Pt wires, respectively. $300 \mu \mathrm{L}$ of the RTIL 1-ethyl-3-methylimidazolium bis(trifluoromethylsulfonyl)imide $\left(\left[\mathrm{C}_{2}\right.\right.$ mim] $\left[\mathrm{NTf}_{2}\right]$, $>99 \%$, Merck, Kilsyth, Victoria, Australia) was contained in a fixed cavity above the change-disk working electrode, consisting of a $1 \mathrm{~cm}$ high polytetrafluoroethylene (PTFE) reservoir custom-made by PINE Research Instrumentation Inc. This RTIL has shown to give chemically reversible HOR peaks previously on Pt surfaces [15]. The electrochemical cell was a modified glass 'T-cell' designed for investigating microsamples of ionic liquids under controlled atmospheres [27, 28], which was purged with $\mathrm{N}_{2}(\geq 99.99 \%$, BOC gases, Welshpool, WA, Australia) to remove impurities (e.g. oxygen, water) naturally present in the RTIL. When the baseline was stable, hydrogen $(99.999 \%$, BOC gases, Welshpool, WA, Australia) was introduced until equilibrium was obtained (typically after 10 minutes). An outlet gas line (PTFE tube) led from the other arm of the cell. All experiments were inside a Faraday cage in a fume cupboard, at $294 \pm 1 \mathrm{~K}$. The gas mixing system (using nitrogen as a dilution gas) is described in [29]. Hydrogen concentration was controlled by varying the relative flow of hydrogen and nitrogen gases, with the total flow rate held constant at $800 \mathrm{sccm}$. Flow rates were varied from 80 to $800 \mathrm{sccm}$ for hydrogen and 720 to $0 \mathrm{sccm}$ for nitrogen. Scanning electron microscopy (SEM) was performed using Zeiss Evo 40XVP model, with an accelerating voltage of $5.0 \mathrm{kV}$. The size of polystyrene spheres, platinum nanoparticles (PtNPs), and macropores was measured using ImageJ. Depth-profiling was performed using a WITec (alpha300 series) confocal microscope. 


\section{Results and Discussion}

\subsection{Electrodeposition and characterisation of electrodes}

Figure 1 shows SEM images of the different modified electrodes. PtNPs were deposited onto GC (Figure 1a) by holding at $-0.2 \mathrm{~V}$ (vs $\mathrm{Ag} / \mathrm{AgCl}$ ) in $5 \mathrm{mM} \mathrm{H}_{2} \mathrm{PtCl}_{6} / 0.5 \mathrm{M} \mathrm{H}_{2} \mathrm{SO}_{4}$ for 20 seconds (charge, $Q=-0.029 \mathrm{Ccm}^{-2}$, averaged over the whole electrode), revealing randomly dispersed nanoparticles of size $215 \pm 14 \mathrm{~nm}$ (averaged over 100 particles, $n=100$ ). The PtNPs will provide the active surface for hydrogen oxidation, since glassy carbon does not have significant activity towards hydrogen.

An image of the polystyrene spheres used for the template on the GC electrode surface (Figure 1d) shows spheres of size $500 \pm 20 \mathrm{~nm}(n=50)$. There are areas of organised hexagonal close packing, with several areas of disorder. This may be due to poor adhesion of the spheres on the surface of the GC electrode, coupled with the relatively fast drying of the casting solvent. More regular alignment was observed on the Pt surface.
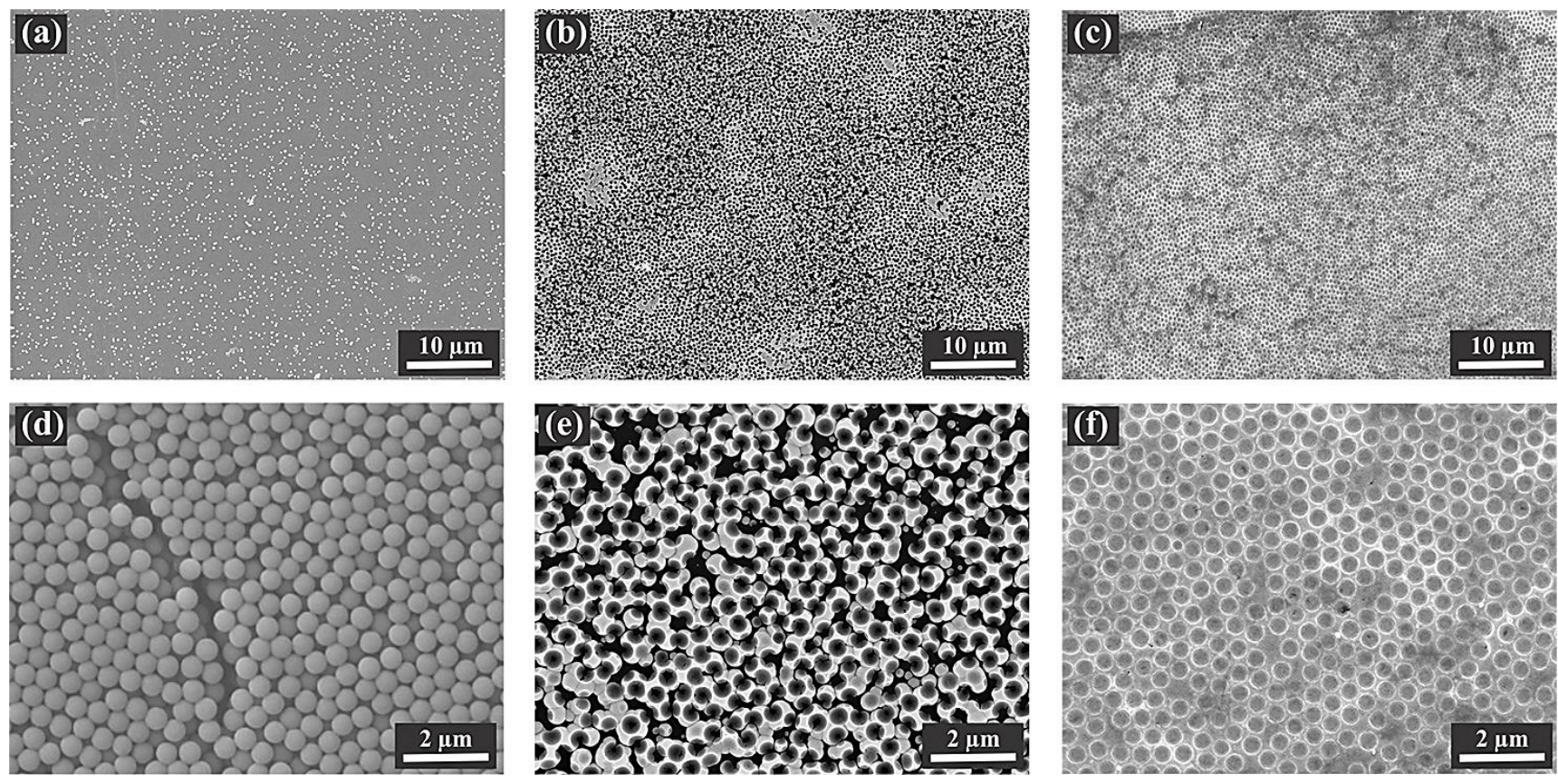

Figure 1. SEM images of (a) Pt nanoparticles on GC, (b) Pt-porous GC, and (c) Pt-porous Pt. Close-up SEM images of (d) polystyrene spheres on GC, (e) Pt-porous GC, and (f) Pt-porous Pt. 
Figures 1b, 1c, 1e and 1f show the polystyrene sphere templated porous-Pt structure, electrodeposited by holding at $-0.2 \mathrm{~V}$ for $300 \mathrm{~s}$ on both $\mathrm{GC}\left(Q=-0.179 \mathrm{Ccm}^{-2}\right)$ and $\mathrm{Pt}\left(Q=-0.180 \mathrm{Ccm}^{-2}\right)$ electrodes at two different magnifications. The height of deposit is relatively regular over the centre of the electrode surface on both electrodes. The thicknesses of the porous-Pt deposits (measured via. depth profiling with a confocal microscope) were ca. $550 \mathrm{~nm}$ and ca. $250 \mathrm{~nm}$ on Pt and GC, respectively - with some evidence of multilayer deposits close to the edges. A thinner deposit on the GC electrode can be attributed to the poorly packed polystyrene spheres. Longer deposition times/charges would likely build up more multilayer deposits [21], but were not required in this work. Pore sizes are $451 \pm 14 \mathrm{~nm}$ ( $n=50)$ on GC, and $440 \pm 12 \mathrm{~nm}(n=50)$ on Pt. The deposit on Pt appears to be strongly bound to the Pt electrode and is fairly uniform over the electrode, but with some obvious nanoporosity when magnified (image not shown). In contrast, the deposit on GC is disconnected, highly cracked, but smoother, and is very easily removed by scratching (although stable for use in the RTIL for hydrogen experiments).

\subsection{Hydrogen oxidation in the RTIL [ $\mathrm{C}_{2} \mathrm{mim}_{[}\left[\mathrm{NTf}_{2}\right]$}

Figure 2 shows cyclic voltammograms $(\mathrm{CVs})$ for the $\mathrm{HOR}$ in $\left[\mathrm{C}_{2} \mathrm{mim}\right]\left[\mathrm{NTf}_{2}\right]$ on the five surfaces. On GC (Figure 2a), there is little response to increasing hydrogen concentration, consistent with hydrogen being relatively inactive on GC. On the four other electrodes, chemically reversible voltammetry was observed. Highly linear plots of peak current vs scan rate indicate adsorption behavior of hydrogen on all electrodes, consistent with that reported previously [14, 17]. Additionally, peak-to-peak separations $\left(\Delta E_{\mathrm{p}}\right)$ increased significantly with increasing scan rate $\left(10-1000 \mathrm{mV} \mathrm{s}^{-1}\right)$, which could be indicative of quasi-reversible kinetics. However, $\Delta E_{\mathrm{p}}$ was observed to also vary with increasing concentration (10 $100 \%$ ) on all surfaces, which may be related to a resistance effect - as verified using the voltametric digital simulation program DigiElch (not shown). The resistance may be a result of the thickness and high viscosity of the electrolyte layer, or due to contact resistance [30] as a result of hydrogen adsorption. It is less likely to be due to diffusion of ions within the porous-Pt or related to the quality 
of the electrical contact of the material, since $\Delta E_{\mathrm{p}}$ also increased on the bare and PtNP modified electrodes.

The major difference between the CVs in Figure 2 is that the current falls almost to zero after the oxidation peak on the porous electrodes, whereas on the Bare Pt and PtNP modified GC electrodes, a more classical peak-shape (typical for a diffusion controlled species) is obtained. This could indicate that hydrogen is strongly-adsorbed in a thin layer within the pores, as similar changes in voltammetric waveshapes were reported by Streeter et al. [31] for electrode surfaces covered by porous layers. This was evident when the system was purged with nitrogen, with ca. 12 hours required for the current to return close to zero, compared to ca. 5 minutes for the non-porous electrodes. It is noted that no-such current drop-off to zero was observed for a standard redox species, ferrocene, on the porous surfaces (not shown), which reveals that the adsorption kinetics of hydrogen at the top layer of the porous material are too slow (at the scan rates used in this study) to enable consumed species to be replenished from the bulk. There is also no evidence of electrogenerated protons being trapped within the film, due to the absence of the proton reduction peak when the cell was flushed with nitrogen after hydrogen oxidation experiments.

Figure 2f shows overlaid CVs for $100 \%$ hydrogen, with an expected concentration (solubility) of ca. $4.2 \mathrm{mM}$ in the RTIL $[12,16,19]$. The widest $\Delta E_{\mathrm{p}}$ was on the bare Pt $(449 \mathrm{mV})$, followed by PtNP GC $(242 \mathrm{mV})>$ Pt-porous Pt $(222 \mathrm{mV})>$ Pt-porous GC $(220 \mathrm{mV})$. This indicates that the kinetics are faster on the electrodeposited materials compared to the polished Pt macrodisk. This could be due to more defect sites on the deposited Pt, which are known points of high electrochemical activity [32]. Peak currents on the porous electrodes are up to double those of the non-porous electrodes, however, the reverse peak currents are similar for all surfaces. The peak current ratio $I_{\mathrm{ox}} / I_{\text {red }}$ is ca. $2-3$ on the porous electrodes compared to $0.7-1$ on the non-porous surfaces, further suggesting a not fully reversible process. Apparent rate constants for electron transfer for hydrogen oxidation $\left(6.5 \times 10^{-3} \mathrm{cms}^{-}\right.$ 
$\left.{ }^{1}\right)$ and proton reduction $\left(4.5 \times 10^{-4} \mathrm{cms}^{-1}\right)$ were reported in this RTIL on polished Pt microdisk electrodes, along with diffusion coefficients for hydrogen $\left(\sim 5.5 \times 10^{-10} \mathrm{~m}^{2} \mathrm{~s}^{-1}\right)$ and the solvated proton $\left(\sim 2.5 \times 10^{-11} \mathrm{~m}^{2} \mathrm{~s}^{-1}\right)[12]$.
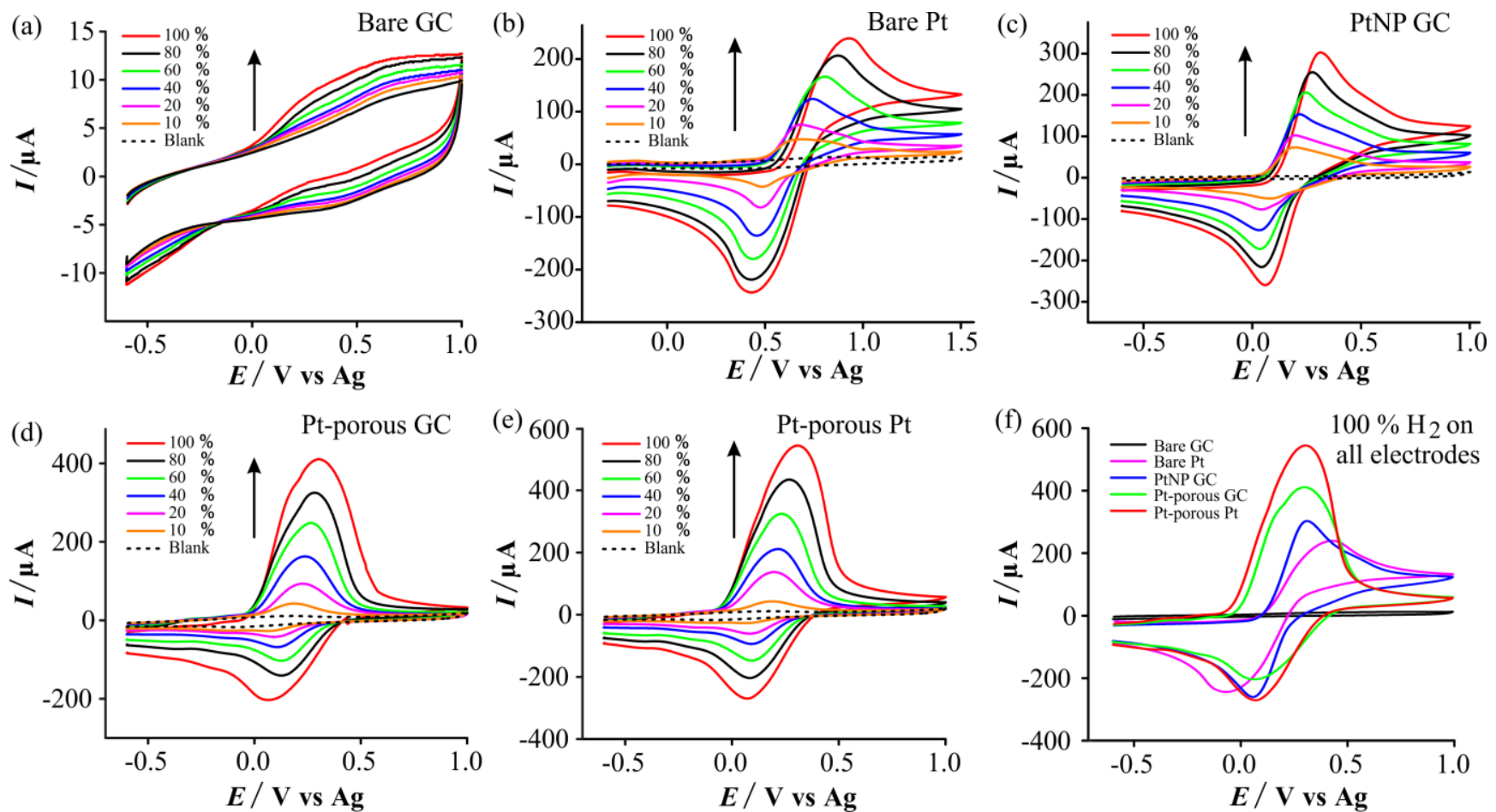

Figure 2. $\mathrm{CV}\left(100 \mathrm{mVs}^{-1}\right)$ for the oxidation of $10-100 \% \mathrm{H}_{2}$ in $\left[\mathrm{C}_{2} \mathrm{mim}\right]\left[\mathrm{NTf}_{2}\right]$ on (a) bare $\mathrm{GC}$, (b) bare Pt, (c) PtNPs GC, (d) Pt-porous GC, (e) Pt-porous Pt, and (f) a comparison of $100 \% \mathrm{H}_{2}$ oxidation on all five electrodes. All CVs were pre-anodized at $+2.0 \mathrm{~V}$ for $60 \mathrm{~s}$ [15]. Note that the CV for bare Pt in (f) has been shifted $-0.3 \mathrm{~V}$ to allow for an easy comparison to the other CVs.

Figure 3 shows a comparison of calibration graphs obtained from the CVs in Figure 2. The negligible sensitivity (gradient) of the bare GC electrode confirms that hydrogen has very little activity on GC. The sensitivity of the other electrodes follow the order: bare Pt (gradient $2.1 \mu \mathrm{A} / \% \mathrm{H}_{2}$ ) $<\mathrm{PtNP}$ GC (gradient $2.5 \mu \mathrm{A} / \% \mathrm{H}_{2}$ ) $<$ Pt-porous GC (gradient $4.0 \mu \mathrm{A} / \% \mathrm{H}_{2}$ ) $<$ Pt-porous Pt (gradient $5.5 \mu \mathrm{A} / \%$ $\mathrm{H}_{2}$ ), showing that the porous electrodes give rise to higher sensitivity - favourable for analytical applications. The higher sensitivity of the porous-Pt on the Pt electrode can be attributed to a larger electroactive surface area, likely due to the greater micro and nano porosity compared to the Pt deposit on the GC electrode - noting that the same deposition parameters were employed, and very similar deposition charges were measured. This suggests that the choice of deposition substrate is crucial to 
ensuring high coverage and ordering of the polystyrene spheres, and maximum porosity of the resultant films. Exploring soft-templated films on cheaper substrate materials (e.g. silver, copper) may be worthwhile in future studies.

Error bars for three repeat measurements on the same electrode are negligible for all surfaces, indicating good reproducibility. However, it should be noted that for the porous materials, the current continued to rise over time while exposed to a fixed $\% \mathrm{H}_{2}$; hence, these calibration graphs were produced using a consistent procedure (applied to all surfaces): (i) starting from low to high concentration, (ii) leaving precisely 5 minutes between concentration changes, and (iii) only recording one CV per concentration. Even steeper gradients were observed if left for a longer gas exposure time, with a plateau current observed at ca. $600 \mu \mathrm{A}$, although the oxidation peak shape remained the same. This suggests an accumulative build-up of $\mathrm{H}_{2}$ gas on the porous material. This behaviour may be problematic for a long-term reversible sensor where accurate quantification is needed, but could be utilised as a highly-sensitivity early-warning leak detector for low concentrations $\left(<0.1 \% \mathrm{H}_{2}\right)$ of hydrogen gas.

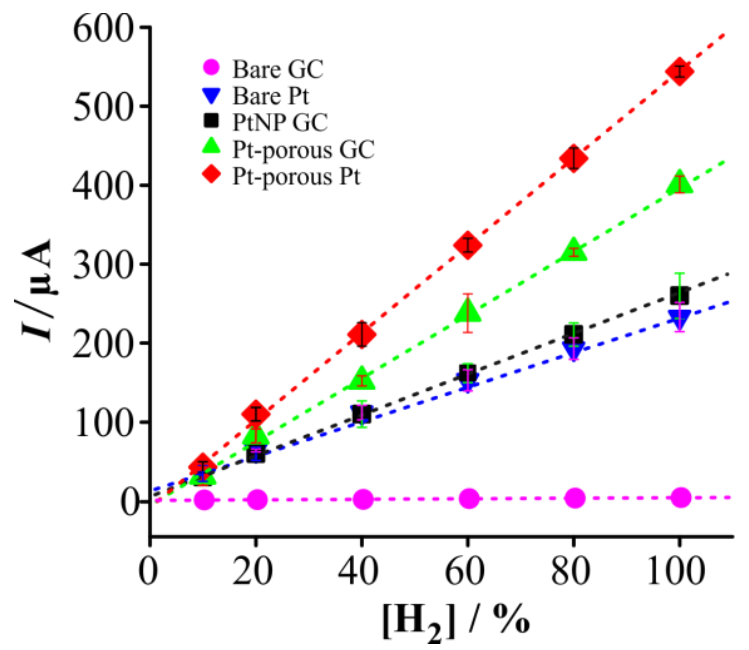

Figure 3. Calibration graphs (peak current vs concentration) for hydrogen oxidation in $\left[\mathrm{C}_{2} \operatorname{mim}\right]\left[\mathrm{NTf}_{2}\right]$ on the five surfaces. Error bars represent one standard deviation for three repeat measurements on the same electrode on different days. 


\section{Conclusions}

Various platinum-modified electrodes have been prepared for the electrochemical detection of hydrogen in an ionic liquid. SEM imaging and depth-profiling of the Pt-porous electrodes revealed that a single layer was deposited, with some multilayers near the edges. Cyclic voltammetry wave shapes suggest strong evidence of hydrogen adsorption and accumulation occurring within the pores, and higher peak currents were found on the porous electrodes - which may allow higher sensitivity for analytical applications. It was observed that hydrogen is retained in the pores and can build-up over a period of time, suggesting that these electrodes may be of potential use as leak detectors for low $\%$ concentrations of hydrogen gas. Future work may include more fundamental studies on the porous materials, especially the effect on diffusion coefficients of species inside the porous structure compared to the bulk.

\section{Acknowledgements}

The authors thank the Australian Research Council for a Discovery grant (DP150101708) and a DECRA Award for DSS (DE120101456). The authors acknowledge the use of equipment and scientific and technical assistance (Ms Elaine Miller) of the Curtin University Electron Microscope Facility, which is partially funded by the University, State and Commonwealth Governments of Australia, and the use of the instruments of the Scanning Probe Microscopy facility at Curtin University, funded by ARC LIEF grant number LE130100121. 


\section{References:}

[1] X. Bévenot, A. Trouillet, C. Veillas, H. Gagnaire, M. Clément, Hydrogen Leak Detection Using an Optical Fibre Sensor for Aerospace Applications, Sens. Act. B: Chemical, 67 (2000) 57-67.

[2] R. Ramachandran, R.K. Menon, An Overview of Industrial Uses of Hydrogen, Int. J. Hydrog. Energy, 23 (1998) 593-598.

[3] J.M. Modak, Haber Process for Ammonia Synthesis, Resonance, (2002) 69-77.

[4] C.S. Huang, T. Kawamura, Y. Toyoda, A. Nakao, Recent Advances in Hydrogen Research as a Therapeutic Medical Gas., Free Radic. Res., 44 (2010) 971-982.

[5] M. Momirlan, T.N. Veziroglu, The Properties of Hydrogen as Fuel Tomorrow in Sustainable Energy System for a Cleaner Planet, Int. J. Hydrog. Energy, 30 (2005) 795-802.

[6] G. Korotcenkov, S.D. Han, J.R. Stetter, Review of Electrochemical Hydrogen Sensors, Chem. Rev., 109 (2009) 1402-1433.

[7] B.E. Conway, D.M. Novak, Oscillatory Kinetics in Electrochemical Oxidation of Hydrogen in an Almost Anhydrous Solvent, J. Phys. Chem., 81 (1977) 1459-1468.

[8] N.M. Marković, B.N. Grgur, P.N. Ross, Temperature-Dependent Hydrogen Electrochemistry on Platinum Low-Index Single-Crystal Surfaces in Acid Solutions, J. Phys. Chem. B, 101 (1997) 54055413.

[9] J.A. Bautista-Martinez, L. Tang, J.-P. Belieres, R. Zeller, C.A. Angell, C. Friesen, Hydrogen Redox in Protic Ionic Liquids and a Direct Measurement of Proton Thermodynamics, J. Phys. Chem. C, 113 (2009) 12586-12593.

[10] S.E. Goodwin, D.A. Walsh, Hydrogen Electrooxidation under Conditions of High Mass Transport in Room-Temperature Ionic Liquids and the Role of Underpotential Deposited Hydrogen, J. Phys. Chem. C, 120 (2016) 11498-11507.

[11] L. Johnson, A. Ejigu, P. Licence, D.A. Walsh, Hydrogen Oxidation and Oxygen Reduction at Platinum in Protic Ionic Liquids, J. Phys. Chem. C, 116 (2012) 18048-18056.

[12] Y. Meng, L. Aldous, R.G. Compton, Electrochemistry of Hydrogen in the Room Temperature Ionic Liquid 1-Butyl-3-methylimidazolium Bis(trifluoromethylsulfonyl)imide: Dissolved Hydrogen “Lubricates” Diffusional Transport, J. Phys. Chem. C, 115 (2011) 14334-14340.

[13] J.B. Rollins, J.C. Conboy, Kinetics and Thermodynamics of Hydrogen Oxidation and Oxygen Reduction in Hydrophobic Room-Temperature Ionic Liquids, J. Electrochem. Soc., 156 (2009) B943-B954.

[14] A.P. Sandoval, M.F. Suarez-Herrera, J.M. Feliu, Hydrogen Redox Reactions in 1-Ethyl-2,3dimethylimidazolium Bis(trifluoromethylsulfonyl)imide on Platinum Single Crystal Electrodes, Electrochem. Commun., 46 (2014) 84-86.

[15] D.S. Silvester, L. Aldous, C. Hardacre, R.G. Compton, An Electrochemical Study of the Oxidation of Hydrogen at Platinum Electrodes in Several Room Temperature Ionic Liquids, J. Phys. Chem. B, 111 (2007) 5000-5007.

[16] D.S. Silvester, K.R. Ward, L. Aldous, C. Hardacre, R.G. Compton, The Electrochemical Oxidation of Hydrogen at Activated Platinum Electrodes in Room Temperature Ionic Liquids as Solvents, J. Electroanal. Chem., 618 (2008) 53-60.

[17] Y. Tang, L. Lin, A. Kumar, M. Guo, M. Sevilla, X. Zeng, Hydrogen Electrooxidation in Ionic Liquids Catalyzed by the NTf2 Radical, J. Phys. Chem. C, 121 (2017) 5161-5167.

[18] Y. Tang, X. Zeng, Electrochemical Oxidation of Hydrogen in Bis(trifluoromethylsulfonyl)imide Ionic Liquids under Anaerobic and Aerobic Conditions, J. Phys. Chem. C, 120 (2016) 23542-23551.

[19] R. Fukuta, Y. Katayama, T. Miura, Hydrogen Electrode Reaction in Some Imide-type Roomtemperature Ionic Liquids, ECS Trans., 3 (2007) 567-576. 
[20] M.E. Abdelsalam, P.N. Bartlett, J.J. Baumberg, S. Coyle, Preparation of Arrays of Isolated Spherical Cavities by Self-Assembly of Polystyrene Spheres on Self-Assembled Pre-patterned Macroporous Films, Adv. Mater., 16 (2004) 90-93.

[21] P.N. Bartlett, J.J. Baumberg, P.R. Birkin, M.A. Ghanem, M.C. Netti, Highly Ordered Macroporous Gold and Platinum Films Formed by Electrochemical Deposition through Templates Assembled from Submicron Diameter Monodisperse Polystyrene Spheres, Chem. Mater., 14 (2002) 2199-2208.

[22] P.N. Bartlett, P.R. Birkin, M.A. Ghanem, Electrochemical Deposition of Macroporous Platinum, Palladium and Cobalt Films Using Polystyrene Latex Sphere Templates, Chem. Commun., (2000) 1671-1672.

[23] L.H.S. Gasparotto, A. Prowald, N. Borisenko, S. Zein El Abedin, A. Garsuch, F. Endres, Electrochemical Synthesis of Macroporous Aluminium Films and their Behavior Towards Lithium Deposition/Stripping, J. Power Sources, 196 (2011) 2879-2883.

[24] B. Kinkhead, J. van Brunen, M.T.Y. Paul, K. Dowling, G. Jerkiewicz, B.D. Gates, Platinum Ordered Porous Electrodes: Developing a Platform for Fundamental Electrochemical Characterization, Electrocatalysis, 4 (2013) 179-186.

[25] M.T.Y. Paul, B. Kinkhead, B.D. Gates, Ordered Porous Gold Electrodes to Enhance the Sensitivity of Enzyme-Based Glucose Sensors, J. Electrochem. Soc., 161 (2014) B3103-B3106.

[26] A. Walcarius, Mesoporous Materials-Based Electrochemical Sensors, Trends Anal. Chem., 27 (2015) 593-603.

[27] U. Schroder, J.D. Wadhawan, R.G. Compton, F. Marken, P.A.Z. Suarez, C.S. Consorti, R.F. de Souza, J. Dupont, Water-Induced Accelerated Ion Diffusion: Voltammetric Studies in 1-Methyl-3[2,6-(S)-dimethylocten-2-yl]imidazolium Tetrafluoroborate, 1-Butyl-3-methylimidazolium Tetrafluoroborate and Hexafluorophosphate Ionic Liquids, New J. Chem., 24 (2000) 1009-1015. [28] D.S. Silvester, A.J. Wain, L. Aldous, C. Hardacre, R.G. Compton, Electrochemical Reduction of Nitrobenzene and 4-Nitrophenol in the Room Temperature Ionic Liquid [C4dmim][N(Tf)2], J. Electroanal. Chem., 596 (2006) 131-140.

[29] J. Lee, K. Murugappan, D.W.M. Arrigan, D.S. Silvester, Oxygen Reduction Voltammetry on Platinum Macrodisk and Screen-Printed Electrodes in Ionic Liquids: Reaction of the Electrogenerated Superoxide Species with Compounds Used in the Paste of Pt Screen-Printed Electrodes?, Electrochim. Acta, 101 (2013) 158-168.

[30] J.H. Singleton, Hydrogen Sorption and the Parahydrogen Conversion of Evaporated Nickel Films, J. Phys. Chem., 60 (1956) 1606-1611.

[31] I. Streeter, G.G. Wildgoose, L. Shao, R.G. Compton, Cyclic voltammetry on electrode surfaces covered with porous layers: An analysis of electron transfer kinetics at single-walled carbon nanotube modified electrodes, Sens. Act. B, 133 (2008) 462-466.

[32] J.C. C., Activated Platinum Surfaces: Electrochemical Studies of Treated Electrodes, Platinum Metals Rev., 8 (1964) 141-142. 